\title{
Current intakes of Interesterified fats in the UK diet and the dietary impact of their substitution with functionally equivalent alternatives
}

\section{Abstract}

Interesterified (IE) saturated-rich fats are widely used to replace partially-hydrogenated fats as hard fats with functional properties needed for spreads/margarines, baked goods, and confectionary, while avoiding the health hazards of trans fats. However, the amount of IE fat consumed and their predicted pubic health impact relative to other application-appropriate fats is unknown. We investigated current intakes of IE fats and the dietary impact of substitution of IE with functionally-equivalent replacement fats using observational data from the UK National Diet and Nutrition Survey (NDNS) (years 5-6 (2012-2014), n =2546) and technical manufacturing data from a UK IE fat supplier. The major sources, types and quantities of IE fat intake were calculated for the total population and different age, ethnic and socioeconomic groups.

IE fat contributes $3.4 \%$ of total fat and $1.1 \%$ of $\%$ total energy intake (TEI) (range $0-10.3 \%$ ), with 19-64 yr old males consuming $1.1 \%$ and females $0.9 \%$ TEI from IE fats. 19-64 yr old quartiles of TEI intake were: $0.1 \%, 0.5 \%, 1.0 \%$ and $2.2 \%$. The $65 \mathrm{yr}+$ age category consumed the most IE fat (1.5\% Energy), followed by $11-18 \mathrm{yr}(1.2 \%)$ and $19-64 \mathrm{yr}(1.0 \%)$. TEI from IE fats was $1 \%$ for high-income households, $1.1 \%$ for the middle-income group and $1.2 \%$ for the low-income group. White individuals consumed more energy from IE fat $(1.2 \% \mathrm{TEI})$ than all other ethnic groups $(0.7 \% \mathrm{TEI})$.

The main sources of IE fats in the UK are from retail spreads (margarines); $54.2 \%$, baked goods; $22.1 \%$, biscuits; $7.7 \%$, non-dairy creams; 5.9\% and confectionery; 5.6\%. Palmitic/lauric acid-rich IE fats, formulated from palm and palm kernel oils, account for 70\% of IE fat consumed. Reformulation of the largest contributor to IE intakes (spreads) to remove IE fat, but retain the same functional characteristics, increased the saturated fatty acid (SFA) composition from $22 \%$ to $37 \%, 25 \%$ to $36 \%, 39 \%$ to $42 \%$ in three most commonly consumed spread formulations. Replacement of IE fats with non-IE alternative formulations would increase population SFA intake by $0.3 \%$ of TEI.

IE fats contribute a small but important amount to the fat intake of the UK population. Removal of IE fat would result in an increase in SFA intake which may have public health implications. Previous research has not focused on the most commonly consumed IE fat blends identified (Palmitic/lauric rich), therefore a re-prioritisation of future research work is recommended to assess the potential impact of IE fats consumed by the UK population.

\section{Conflict of Interest}

Jo Bruce is employed by ADM, a manufacturer of edible oils and fats 